\title{
Gradient-Based Approach to Solve Optimal Periodic Output Feedback Control Problems*
}

\author{
ANDRAS VARGA, and STEFAN PIETERS ${ }^{\dagger}$
}

Appeared in Automatica

Vol. 34, No. 4, pp.477-481, 1998

Key Words - Periodic systems, periodic output feedback, periodic Lyapunov equation, computational methods.

\begin{abstract}
We discuss the numerical solution of the output feedback optimal periodic control problem by using a gradient search based optimization approach. Extension of this approach to the simultaneous stabilization of periodic multimodels is also considered. For the evaluation of function and its gradient explicit expressions are derived which involve the numerical solution of a pair of discrete-time periodic Lyapunov equations. Numerically reliable algorithms based on the periodic Schur decomposition are used for the efficient solution of these equations. A satellite positioning example illustrates the effectiveness of the presented approach to solve a practical periodic control problem.
\end{abstract}

*A short version of this paper has been presented at the CACSD'96 Symp., Dearborn, MI, Sept. 15-18, 1996

${ }^{\dagger}$ The authors are with DLR - Oberpfaffenhofen, German Aerospace Research Establishment, Institute of Robotics and System Dynamics, P.O.B. 1116, D-82230 Wessling, Germany;Phone: +49-8153-282408, Fax:+498153-1441, E-mail: Andreas.Varga@dlr.de. 


\section{Introduction}

The static output feedback control problem received considerable attention in the control literature; see for instance the survey papers of Mäkilä and Toivonen (1987) and Syrmos et al. (1997) and the references therein. Although the fundamental question of existence of stabilizing output feedback controllers is still open, a tremendous effort has been invested towards developing computational approaches to solve this problem if a solution exists. One of the popular design approaches of static output feedback controllers is the solution of a linear-quadratic (LQ) optimization problem analogously to the case of full state feedback.

There are basically two computational approaches, both iterative, for the solution of the optimal LQ output feedback problem. The first approach, pioneered for continuous-time systems by Levine and Athans (1970), attempts to solve the nonlinear matrix equations expressing the necessary conditions for the optimality; see (Mäkilä and Toivonen; 1987) for a survey of computational methods. This approach has been extended to periodic systems with constant static output feedback by Broussard and Halyo (1984). It is commonly accepted that the algorithms for this approach are generally inferior, with respect to their convergence properties, to those for the second approach, where nonlinear programming descent methods are used to perform the minimization of the quadratic cost function. To solve the unconstrained minimization problem, both general purpose methods, as the popular conjugate gradient or BFGS quasi-Newton methods, as well as specialized gradient methods, as the enhanced descent Anderson-Moore method (Mäkilä and Toivonen; 1987), have been employed. All these methods rely on proven convergence results which, under standard assumptions, guarantee the convergence to a local minimum of the performance index. The availability of efficient software implementations of several unconstrained minimization algorithms, moved the focus in solving optimal LQ output feedback problems from finding convergent minimization algorithms to the derivation of efficient numerical evaluation schemes of the cost functionals and gradients for various problems, as for instance, the computational solution proposed by Hyslop et al. (1992) to solve periodic output feedback control problems employing multiple input sampling.

In the last few years there has been a constantly increasing interest for the development of numerical algorithms for the analysis and design of linear periodic discrete-time control systems (Bittanti et al.; 1988; Hench and Laub; 1994; Sreedhar and Van Dooren; 1993; Aeyels and Willems; 1995). In this paper we discuss the numerical solution of the optimal LQ periodic output feedback control problem by using a gradient search based optimization approach. An extension involving the simultaneous optimization and stabilization of periodic multimodels is also considered. For the evaluation of cost functions and their gradients explicit expressions are derived which involve the numerical solution of a pair of discrete-time periodic Lyapunov equations. Efficient numerically reliable algorithms based on periodic Schur decomposition are used for the solution of these equations (Varga; 1997). A satellite positioning example illustrates the effectiveness of the proposed approach to solve a practical periodic output feedback problem.

Notations and notational conventions. For a periodic square time-varying matrix $A_{k}$ of integer period $K$ we denote $\Phi_{A}(j, i)=A_{j-1} A_{j-2} \cdots A_{i}$ for $j>i$ and $\Phi_{A}(i, i):=I$. The matrix $\Phi_{A}(\tau+K, \tau)$ is called the monodromy matrix at time $\tau$ and its eigenvalues, independent of $\tau$, are called the characteristic multipliers. For a periodic matrix $X_{k}$ of period $K$ we use alternatively the script notation $\mathcal{X}$ which associates the block-diagonal matrix $\mathcal{X}=\operatorname{diag}\left(X_{0}, X_{1}, \ldots, X_{K-1}\right)$ to the cyclic sequence of matrices $X_{k}, k=0, \ldots, K-1$. This notation is consistent with the standard matrix operations with block-diagonal matrices. We denote with $\sigma \mathcal{X}$ the $K$-cyclic shift $\sigma \mathcal{X}=\operatorname{diag}\left(X_{1}, \ldots, X_{K-1}, X_{0}\right)$ applied to the cyclic sequence $X_{k}, k=0, \ldots, K-1$. 


\section{Optimal LQ Periodic Output Feedback}

Consider the linear discrete-time periodic system of the form

$$
\begin{aligned}
x_{k+1} & =A_{k} x_{k}+B_{k} u_{k} \\
y_{k} & =C_{k} x_{k}
\end{aligned}
$$

where the matrices $A_{k} \in \mathrm{R}^{n \times n}, B_{k} \in \mathrm{R}^{n \times m}$, and $C_{k} \in \mathrm{R}^{p \times n}$ are periodic with period $K \geq 1$. Such models arise usually from the discretization of linear continuous-time periodic models which are the primary mathematical descriptions encountered in most practical applications. Let $J$ be a quadratic performance index of the form

$$
J=E\left\{\sum_{k=0}^{\infty}\left[x_{k}^{T} Q_{k} x_{k}+u_{k}^{T} R_{k} u_{k}\right]\right\}
$$

where $Q_{k} \geq 0$ and $R_{k}>0$, for $k=0, \ldots, K-1$ are symmetric periodic matrices of period $K$. The expected values are used in the performance index (2) to take into account the dependence of $J$ of the initial conditions. We shall assume that $x_{0}$ is a random variable with zero mean and known covariance $X_{0}=E\left\{x_{0} x_{0}^{T}\right\}$.

In this section we address the problem to determine the optimal LQ periodic output-feedback control law

$$
u_{k}^{*}=F_{k} y_{k}
$$

which minimizes the performance index (2), while stabilizing the closed-loop system. Conditions for the existence and global uniqueness of the solution to the above problem are not known even for the non-periodic case (Syrmos et al.; 1997). A straightforward necessary condition for the existence of a minimizing periodic gain matrix is the periodic output stabilizability of the system (1); that is, there exists a stabilizing periodic output feedback matrix $F_{k}$ such that $\rho\left(\Phi_{\bar{A}}(K, 0)\right)<1$, where $\bar{A}_{k}=A_{k}+B_{k} F_{k} C_{k}$ and $\rho(\cdot)$ is the spectral radius of a square matrix. Note however, that there may be more than one local minimum, so that generally the minimization of $J$, by using for example nonlinear programming techniques, may not lead to the global minimum. In what follows we assume that a stabilizing periodic output feedback matrix for the system (1) exists.

For the solution of the optimal LQ periodic output feedback problem in general no closed form solutions can be found even for standard state space systems. Thus iterative search methods must be used to compute the optimizing periodic output feedback matrix. Provided we use general purpose unconstrained nonlinear programming methods based on gradient techniques, it is necessary to evaluate for a given stabilizing periodic output feedback $F_{k}$ the corresponding values of the cost functional (2) and of its gradient with respect to $F_{k}$. The expressions of function and gradient can be computed on the basis of the following result.

Proposition 1. Let $F_{k}$ be a stabilizing periodic output feedback gain and let denote $\overline{\mathcal{A}}=\mathcal{A}+$ $\mathcal{B F C}, \overline{\mathcal{Q}}=\mathcal{Q}+\mathcal{C}^{T} \mathcal{F}^{T} \mathcal{R} \mathcal{F C}$, and $\mathcal{G}=\operatorname{diag}\left(0,0, \ldots, X_{0}\right)$. Then the expressions for the function (2) and its gradient are:

$$
\begin{aligned}
J(\mathcal{F}) & =\operatorname{tr}(\sigma \mathcal{P G}) \\
\nabla_{\mathcal{F}} J(\mathcal{F}) & =2\left(\mathcal{R} \mathcal{F} \mathcal{C}+\mathcal{B}^{T} \sigma \mathcal{P} \overline{\mathcal{A}}\right) \mathcal{S C}^{T}
\end{aligned}
$$

where $\mathcal{P}$ and $\mathcal{S}$ satisfy the discrete periodic Lyapunov equations (DPLEs):

$$
\begin{aligned}
\mathcal{P} & =\overline{\mathcal{A}}^{T} \sigma \mathcal{P} \overline{\mathcal{A}}+\overline{\mathcal{Q}} \\
\sigma \mathcal{S} & =\overline{\mathcal{A}} \mathcal{S} \overline{\mathcal{A}}^{T}+\mathcal{G} .
\end{aligned}
$$


Proof. See Appendix.

The above formulas can be also employed to derive the expressions of the function and its gradient in the case of a constant output feedback.

Corollary 1. Let $F$ be a constant stabilizing output feedback matrix and let denote $\bar{A}_{k}=A_{k}+$ $B_{k} F C_{k}$ and $\bar{Q}_{k}=Q_{k}+C_{k}^{T} F^{T} R_{k} F C_{k}$. Then the expressions for the function (2) and its gradient are:

$$
\begin{aligned}
J(F) & =\operatorname{tr}\left(P_{0} X_{0}\right) \\
\nabla_{F} J(F) & =2 \sum_{j=0}^{K-1}\left(R_{j} F C_{j}+B_{j}^{T} P_{j+1} \overline{A_{j}}\right) S_{j} C_{j}^{T}
\end{aligned}
$$

where $\mathcal{P}$ and $\mathcal{S}$ satisfy the DPLEs (4) and (5), respectively.

Having explicit analytical expressions for the function and its gradient, it is easy to employ any gradient based technique to minimize $J$, provided an initial stabilizing output feedback gain is available. Because of the potentially high dimension of the minimization problem, unconstrained descent methods like the limited memory BFGS method (Liu and Nocedal; 1989) used in conjunction with an inexact line search procedure with guaranteed decrease (Moré and Thuente; 1994) are especially well suited. These methods are implemented within the MINPACK-2 project (the successor of MINPACK-1; see Moré; 1980), offering a convenient reverse communication interface which allows an easy implementation of function and gradient computations. These codes are very flexible, allowing to avoid the generation of destabilizing feedback matrices during the iteration steps.

To use gradient search methods, an important subproblem in solving an optimal output feedback control problem is the initialization of the search process in the case of an unstable system. An initial stabilizing output feedback gain can be computed in several ways (see Mäkilä and Toivonen; 1987). Probably the simplest and most convenient approach is to use the minimization procedure itself to find a stabilizing feedback. This can be done by solving a sequence of modified problems, which finally leads to a stabilizing gain matrix if one exists. We can solve the optimal output feedback problem repeatedly for modified systems with the pair $\left(A_{0}, B_{0}\right)$ replaced by scaled pairs of the form $\left(\alpha_{j} A_{0}, \alpha_{j} B_{0}\right)$. To determine the optimal solution $F_{k}^{(j)}$ corresponding to a modified problem, the positive scaling $\alpha_{j}$ is chosen such that $\alpha_{j} \rho\left(\Phi_{A+B F^{(j-1)} C}(K, 0)\right)<1$. To start the iterations, we choose $\alpha_{1}$ such that $F_{k}^{(0)}=0$ is a trivial initialization. Because the sequence of positive numbers $\left\{\alpha_{j}\right\}$ is strictly increasing, a stabilizing output gain eventually results when $\alpha_{j} \geq 1$ for a certain $j$. This technique can even be used to achieve a prescribed stability degree for the closed-loop system. To improve the efficiency of the preliminary stabilization, it is recommendable to perform the necessary optimizations by using larger tolerances in the stopping criterion of the minimizer. Only the final step in determining the optimal output feedback needs to be performed using the desired tolerance (set by the user).

It is interesting to note that the gradient approach allows to cope easily with structured feedback gain matrices. How structure enters in computations has been shown by Toivonen and Mäkilä (1985) in the context of optimal decentralized control. By assuming that some elements of $F_{k}$ are fixed (for instance set to zero), we can solve the output feedback optimization problem by using straightforward mapping mechanisms to extract the active set of components of $F_{k}$ and of the gradient $\nabla_{F_{k}} J\left(F_{k}\right)$ before passing them to the minimization routine. Notice that although the parameter search is performed on a reduced set of elements of $F$, the expressions of function and gradient are still valid.

The instantaneous (or memoryless) periodic output feedback approach using the optimal output feedback controller (3) can be employed to solve various particular periodic control problems arising from multirate sampled-data control problems. Several multirate control schemes, as the 
multiple input sampling (Chammas and Leondes; 1978; Hyslop et al.; 1992), multirate input sampling (Araki and Hagiwara; 1988) or the multirate output sampling (Hagiwara and Araki; 1988) can be easily turned into memoryless periodic output feedback problems and solved by the proposed approach.

\section{Robust LQ Periodic Output Feedback Control}

We can formulate, similarly to the case of standard systems (Mäkilä and Toivonen; 1987), the problem of finding a fixed periodic feedback gain to obtain satisfactory performance over a set of operating points. Let $N$ periodic systems be defined by the periodic matrix triples $\left(A_{k}^{i}, B_{k}^{i}, C_{k}^{i}\right)$, $i=1, \ldots, N$. These $N$ systems can be seen as a multimodel describing the same physical system in different operating conditions and/or configurations (describing for instance various sensor/actuator failure situations). Let $J^{i}, i=1, \ldots, N$ be the quadratic performance indices of the form (2) defined by the set of periodic weighting matrix pairs $\left(Q_{k}^{i}, R_{k}^{i}\right), i=1, \ldots, N$. We assume that the initial state covariances $X_{0}^{i}, i=1, \ldots, N$, are known.

The simultaneous optimization and stabilization of the $N$ periodic systems with a unique periodic output feedback $F_{k}$ can be formulated as a large order decentralized periodic output feedback control problem, with the system state, input and output vectors formed by stacking the corresponding vectors of the $N$ systems and with a decentralized output feedback matrix of a block-diagonal form having all diagonal blocks equal to $F_{k}$. To perform the simultaneous optimization of the $N$ systems we can use for instance an aggregated performance index of the form

$$
J_{R}=\sum_{i=1}^{N} w_{i} J_{i}
$$

where $w_{i}>0, i=1, \ldots, N$ are appropriate weights satisfying $\sum_{i=1}^{N} w_{i}=1$.

To minimize the performance index (6) by using gradient based search methods, we need expressions to efficiently evaluate the performance index and its gradient. By using the script notation, the following result generalizes Proposition 1 for the case of multimodels.

Proposition 2. Let $F_{k}$ be a periodic output feedback gain which simultaneously stabilizes all $N$ periodic systems. Let denote $\overline{\mathcal{A}^{i}}=\mathcal{A}^{i}+\mathcal{B}^{i} \mathcal{F} \mathcal{C}^{i}, \overline{\mathcal{Q}^{i}}=\mathcal{Q}^{i}+\mathcal{C}^{i T} \mathcal{F}^{T} \mathcal{R}^{i} \mathcal{F} \mathcal{C}^{i}$, and $\mathcal{G}^{i}=$ $\operatorname{diag}\left(0,0, \ldots, X_{0}^{i}\right)$. Then the expressions for the function (6) and its gradient are:

$$
\begin{aligned}
J(\mathcal{F}) & =\sum_{i=1}^{N} w_{i} \operatorname{tr}\left(\sigma \mathcal{P}^{i} \mathcal{G}^{i}\right) \\
\nabla_{\mathcal{F}} J(\mathcal{F}) & =2 \sum_{i=1}^{N} w_{i}\left(\mathcal{R}^{i} \mathcal{F} \mathcal{C}^{i}+\mathcal{B}^{i T} \sigma \mathcal{P}^{i} \overline{\mathcal{A}^{i}}\right) \mathcal{S}^{i} \mathcal{C}^{i T}
\end{aligned}
$$

where $\mathcal{P}^{i}$ and $\mathcal{S}^{i}$, for $i=1, \ldots, N$, satisfy the DPLEs:

$$
\mathcal{P}^{i}={\overline{\mathcal{A}^{i}}}^{T} \sigma \mathcal{P}^{i} \overline{\mathcal{A}^{i}}+\overline{\mathcal{Q}^{i}}
$$

and

$$
\sigma \mathcal{S}^{i}=\overline{\mathcal{A}^{i}} \mathcal{S}^{i} \overline{\mathcal{A}}^{T}+\mathcal{G}^{i}
$$

respectively.

The increased stabilization potential of the periodic output feedback applied to multimodels has been shown by Kabamba (1987). Thus, a very promising application of the optimal periodic output feedback approach is to use a single periodic time-varying output feedback controller to simultaneously stabilize several time-invariant systems. Note that the proposed memoryless output feedback approach can be used to solve also multimodel control problems employing multiple input sampling schemes as those proposed in (Kabamba; 1987). 


\section{Numerical evaluation of function and gradient}

Each evaluation of the performance index (2) and its gradient involves (see Proposition 1) the solution of a pair of DPLEs: a reverse time discrete periodic Lyapunov equation of the form

$$
\mathcal{P}=\mathcal{A}^{T} \sigma \mathcal{P} \mathcal{A}+\mathcal{Q}
$$

and a dual forward time discrete periodic Lyapunov equation of the form

$$
\sigma \mathcal{S}=\mathcal{A S} \mathcal{A}^{T}+\mathcal{G}
$$

where all characteristic values of the monodromy matrix $\Phi_{A}(K, 0)$ lie in the unit circle of the complex plane. This ensures the existence of a unique solution of both equations. Note that for standard systems these are two discrete Lyapunov equations which can be solved efficiently with a computational cost which is marginally greater than the cost of solving a single Lyapunov equation. The preservation of this feature is even more stringent for the periodic case, because of the much higher computational effort involved in solving a single periodic Lyapunov equation. Fortunately, this goal can be achieved with the recently proposed numerically stable algorithms to solve DPLEs (Varga; 1997).

The algorithms proposed in (Varga; 1997) resemble the method of Bartels and Stewart (1972) and rely on an initial reduction of the Lyapunov equation to a simpler form by using the periodic Schur decomposition (PSD) of a matrix product (Bojanczyk et al.; 1992; Hench and Laub; 1994). According to Bojanczyk et al. (1992), given the matrices $A_{k}, k=0,1, \ldots, K-1$, there exist orthogonal matrices $Z_{k}, k=0,1, \ldots, K-1$ such that $\widetilde{A}_{K-1}=Z_{0}^{T} A_{K-1} Z_{K-1}$ is in a real Schur form and the matrices $\widetilde{A}_{k}=Z_{k+1}^{T} A_{k} Z_{k}$ for $k=0, \ldots, K-2$ are upper triangular. It follows that the product $\widetilde{A}_{K-1} \cdots \widetilde{A}_{0}$ is in a real Schur form, but the PSD can be determined without explicitly forming this product. The transformation to compute the PSD can be expressed using the script notation as $\widetilde{\mathcal{A}}=\sigma \mathcal{Z}^{T} \mathcal{A Z}$. By using this transformation we can simplify the solution of the two DPLEs as shown in the procedure given below:

1. Compute the orthogonal $\mathcal{Z}$ to reduce $\Phi_{A}(K, 0)$ to the PSD.

2. Compute $\widetilde{\mathcal{A}}=\sigma \mathcal{Z}^{T} \mathcal{A Z}, \widetilde{\mathcal{Q}}=\mathcal{Z}^{T} \mathcal{Q Z}$ and $\widetilde{\mathcal{G}}=\sigma \mathcal{Z}^{T} \mathcal{G} \sigma \mathcal{Z}$.

3. Solve the reduced DPLEs $\widetilde{\mathcal{P}}=\widetilde{\mathcal{A}}^{T} \sigma \widetilde{\mathcal{P}} \widetilde{\mathcal{A}}+\widetilde{\mathcal{W}}$ and $\sigma \widetilde{\mathcal{S}}=\widetilde{\mathcal{A}} \widetilde{\mathcal{A}} \widetilde{\mathcal{A}}^{T}+\widetilde{\mathcal{G}}$.

4. Compute $\mathcal{P}=\mathcal{Z} \widetilde{\mathcal{P}} \mathcal{Z}^{T}$ and $\mathcal{S}=\mathcal{Z} \widetilde{\mathcal{S}} \mathcal{Z}^{T}$.

Note that only one computation of the PSD is necessary to solve both DPLEs. The reduced DPLEs are solved by using special forward and backward substitution algorithms; for details see (Varga; 1997). The number of floating-point operations (flops) necessary to solve the pair of DPLEs is roughly $N_{P S D}+7 K n^{3}$, where $N_{P S D} \approx 25 K n^{3}$ is the number of flops to determine the PSD.

\section{Example}

Consider the optimal periodic output feedback control of a spacecraft pointing and attitude system as described by Pittelkau (1993). The continuous-time linearized state space model of 
the spacecraft system is described by the matrices

$$
\begin{aligned}
& A=\left[\begin{array}{rrrr}
0 & 0 & 0.05318064 & 0 \\
0 & 0 & 0 & 0.05318064 \\
-0.001352134 & 0 & 0 & -0.07099273 \\
0 & -0.0007557182 & 0.03781555 & 0
\end{array}\right], \\
& B(t)=\left[\begin{array}{c}
0 \\
0 \\
0.1389735 \cdot 10^{-6} \sin \left(\omega_{0} t\right) \\
-0.3701336 \cdot 10^{-7} \cos \left(\omega_{0} t\right)
\end{array}\right], \quad C=\left[\begin{array}{llll}
1 & 0 & 0 & 0 \\
0 & 1 & 0 & 0
\end{array}\right],
\end{aligned}
$$

where $\omega_{0}=0.00103448 \mathrm{rad} / \mathrm{s}$ is the orbital frequency. Notice that $A$ is a constant matrix with all its eigenvalues on the imaginary axis. The matrix $B(t)$ is however a time-dependent periodic matrix with the period $2 \pi / \omega_{0}$. The components of the state vector are the roll and yaw angles and their derivatives, the control input is the pitch magnetic moment produced by magnetic torquer bars, and the measured outputs are the roll and yaw angles. The control effect is generated by the interaction between a magnetic actuator and the Earth's magnetic field. The control problem is to determine a stabilizing output feedback control law which ensures a stable operation of the spacecraft on the orbit.

A continuous-time approach to solve the control problem is rather involved because of inherent mathematical and computational difficulties. However a discrete-time approach allows a relatively easy solution of the problem by using the computational method developed in this paper. Let $K$ denote the number of sampling periods to generate control effects in a complete orbit and let $T$ be the corresponding sampling period $T=2 \pi /\left(\omega_{0} K\right)$. The matrices of the discrete-time periodic system can be computed explicitly as $A_{k}=\exp (A T), B_{k}=\int_{k T}^{(k+1) T} e^{[A(k+1) T-\tau]} B(\tau) d \tau$. To investigate the performance of the output feedback control for different values of $K$, we generated discrete-time models for $K=10,20,40$ and 120. For reference purposes we give the matrices of the discretized periodic model which results for $K=120$ and $T=50.61468$ sec:

$$
\begin{gathered}
A_{k}=\left[\begin{array}{rrrr}
0.9506860 & 0.0429866 & 0.4827320 & -2.5564383 \\
-0.0409684 & 0.9721628 & 1.3617328 & 0.5081454 \\
-0.0122736 & 0.0363280 & -0.8671394 & -0.6014295 \\
-0.0346225 & -0.0072209 & 0.3203622 & -0.8456626
\end{array}\right], \\
B_{k}=10^{-5} \cdot\left[\begin{array}{r}
0.2220925 \\
-0.1300536 \\
0.1877217 \\
-0.0271167
\end{array}\right] \cos \left(\omega_{0} k T\right)+10^{-5} \cdot\left[\begin{array}{l}
0.5035620 \\
0.4241087 \\
0.1218290 \\
0.3583826
\end{array}\right] \sin \left(\omega_{0} k T\right) .
\end{gathered}
$$

For different values of $K$ we computed the stabilizing periodic output feedback $F_{k}$ which minimizes the performance index $(2)$, where $Q_{k}=\operatorname{diag}(2,1,0,0)$ and $R_{k}=10^{-11}$. In the following table, for each value of $K$ we included the achieved spectral radius of the closed-loop monodromy matrix and the corresponding value of the performance index.

\begin{tabular}{|c||c|c|r|r|}
\hline$K$ & 10 & 20 & \multicolumn{1}{|c|}{40} & \multicolumn{1}{|c|}{120} \\
\hline$\rho\left(\Phi_{A+B F C}(K, 0)\right)$ & 0.967 & 0.944 & 0.163 & 0.00036 \\
$J(\mathcal{F})$ & 593.2 & 577.8 & 62.4 & 59.65 \\
\hline
\end{tabular}

It can be seen that for $K=120$, the dynamical performance of the closed-loop system, expressed in terms of the spectral radius of the monodromy matrix, is completely satisfactory. The practical 
implementation of the control algorithm requires the storage of only $1202 \times 1$ gain matrix values. In comparison, by using the continuous-time approach of Pittelkau (1993), 1000 sample values for a 4 by 1 state-feedback controller gain matrix are stored and linear interpolation is used to compute the gain values between samples. Additionally, a constant state estimator was used to estimate the state vector. Thus for all practical purposes the periodic output feedback approach is a completely satisfactory alternative to existing spacecraft attitude control techniques.

The optimization problem for $K=120$ is of dimension 240 and has been solved using the limited memory BFGS algorithm. In the following table we give some results illustrating the performances of the optimization algorithm for different relative tolerances RELTOL used in the termination criteria. The figures for the number of iterations $N_{I T}$, the number of function/gradient evaluations $N_{F / G}$, and the CPU times (in seconds) correspond to starting the iterations from a previously computed stabilizing periodic feedback $\mathcal{F}_{0}$ at which $J\left(\mathcal{F}_{0}\right)=38527$. The computations have been done on a 100 Mhz HP-9000/715 workstation running under HPUX 10.2.

\begin{tabular}{|l||r|r|r|r|r|r|}
\hline RELTOL & $10^{-2}$ & $10^{-3}$ & $10^{-4}$ & $10^{-5}$ & $10^{-6}$ & $10^{-7}$ \\
\hline$N_{I T}$ & 33 & 50 & 100 & 178 & 328 & 966 \\
$N_{F / G}$ & 44 & 66 & 121 & 213 & 377 & 1088 \\
$J(\mathcal{F})$ & 90.9 & 68.5 & 63.2 & 61.6 & 60.7 & 59.9 \\
CPU time & 16 & 23 & 42 & 74 & 130 & 377 \\
\hline
\end{tabular}

The CPU time for a function/gradient evaluation amounts to a mean value of about 0.35 seconds.

\section{Conclusion}

A numerical approach to solve the optimal periodic output feedback control problem has been developed. Formulas for explicit evaluation of the function and its gradient have been derived. Each function/gradient evaluation involves the solution of two DPLEs which can be solved by recently developed numerically reliable computational algorithms. A set of LAPACK based Fortran routines have been implemented to compute the PSD and to solve four types of periodic Lyapunov equations. A satellite attitude control problem has been solved to illustrate the proposed computational approach.

\section{References}

Aeyels, D. and Willems, J. L. (1995). Pole assignment for linear periodic systems by memoryless output feedback, IEEE Trans. Autom. Control 40: 735-739.

Araki, M. and Hagiwara, T. (1988). Pole assignment by multirate sampled-data, Int. J. Control 44: 1661-1673.

Bartels, R. H. and Stewart, G. W. (1972). Algorithm 432: Solution of the matrix equation $\mathrm{AX}+\mathrm{XB}=\mathrm{C}$, Comm. ACM 15: 820-826.

Berger, C. S. (1976). The derivatives of useful functions in control theory, Int. J. Control 24: 431-433.

Bittanti, S., Colaneri, P. and Nicolao, G. D. (1988). The difference periodic Riccati equation for the periodic prediction problem, IEEE Trans. Autom. Control 33: 706-712. 
Bojanczyk, A. W., Golub, G. and Van Dooren, P. (1992). The periodic Schur decomposition. Algorithms and applications, in F. T. Luk (ed.), Proceedings SPIE Conference, Vol. 1770, pp. 31-42.

Broussard, J. R. and Halyo, N. (1984). Optimal multi-rate output feedback, Proc. 23rd Conference Decision and Control, Las Vegas, NV, pp. 926-929.

Chammas, A. B. and Leondes, C. T. (1978). On the design of linear time-invariant systems by periodic output feedback. Part I. Discrete-time pole assignment, Int. J. Control 27: 885-894.

Hagiwara, T. and Araki, M. (1988). Design of a stable state feedback controller based on the multirate sampling of the plant output, IEEE Trans. Autom. Control 33: 821-819.

Hench, J. J. and Laub, A. J. (1994). Numerical solution of the discrete-time periodic Riccati equation, IEEE Trans. Autom. Control 39: 1197-1210.

Hyslop, G. L., Schättler, H. and Tarn, T.-J. (1992). Descent algorithms for optimal periodic output feedback control, IEEE Trans. Autom. Control 37: 1893-1904.

Kabamba, P. T. (1987). Control of linear systems using generalized sampled-data hold functions, IEEE Trans. Autom. Control 32: 772-783.

Levine, W. S. and Athans, M. (1970). On the determination of the optimal constant output feedback gains for linear multivariable systems, IEEE Trans. Autom. Control 15: 44-48.

Liu, D. C. and Nocedal, J. (1989). On the limited memory BFGS method for large scale optimization, Mathematical Programming 45: 503-528.

Mäkilä, P. M. and Toivonen, H. T. (1987). Computational methods for parametric LQ problems - a survey, IEEE Trans. Autom. Control 32: 658-671.

Moré, J. J. (1980). User's Guide for MINPACK-1, Applied Mathematics Division Report ANL80-74, Argonne National Laboratory, Argone,IL.

Moré, J. J. and Thuente, D. J. (1994). On line search algorithms with guaranteed sufficient decrease, ACM Trans. Math. Software 20: 286-307.

Pieters, S. (1995). Gradient methods for the computation of the LQ optimal output feedback, Technical Report TR R186-95, DLR-Oberpfaffenhofen, German Aerospace Research Establishment, Istitute for Robotics and System Dynamics.

Pittelkau, M. E. (1993). Optimal periodic control for spacecraft pointing and attitude determination, J. of Guidance, Control, and Dynamics 16: 1078-1084.

Sreedhar, J. and Van Dooren, P. (1993). Periodic Schur form and some matrix equations, in U. Helmke, R. Mennicken and J. Saurer (eds), Proc. MTNS'93, Regensburg, Germany, Vol. I, pp. 339-362.

Syrmos, V. L., Abdallah, C. T., Dorato, P. and Grigoriadis, K. (1997). Static output feedback: A survey, Automatica 33: 125-137.

Toivonen, H. T. and Mäkilä, P. M. (1985). A descent Anderson-Moore algorithm for optimal decentralized control, Automatica 21: 743-744.

Varga, A. (1997). Periodic Lyapunov equations: some applications and new algorithms, Int. J. Control. 


\section{Appendix.}

In the proof of Proposition 1 we employ some standard formulas for gradients (Pieters; 1995). These formulas are summarized in the following two lemmas. Lemma 2 is based on the derivation method employed by Berger (1976).

Lemma 1. Let $J_{1}(F)=\operatorname{tr}(X F Y), J_{2}(F)=\operatorname{tr}\left(X F^{T} Y\right)$ and $J_{3}(F)=\operatorname{tr}\left(X F^{T} Y F Z\right)$ be scalar functions of the matrix variable $F$, where $X, Y$ and $Z$ are matrices which do not depend on $F$. Then

$$
\begin{aligned}
\nabla_{F} J_{1}(F) & =\frac{\partial}{\partial F} \operatorname{tr}(X F Y)=X^{T} Y^{T} \\
\nabla_{F} J_{2}(F) & =\frac{\partial}{\partial F} \operatorname{tr}\left(X F^{T} Y\right)=Y X \\
\nabla_{F} J_{3}(F) & =\frac{\partial}{\partial F} \operatorname{tr}\left(X F^{T} Y F Z\right)=Y F Z X+Y^{T} F X^{T} Z^{T} .
\end{aligned}
$$

Lemma 2. Let $J(F)=\operatorname{tr}(P X)$ be a scalar function of the matrix variable $F$, where the symmetric matrix $X$ does not depend on $F$ and $P=P(F)$ satisfies the discrete Lyapunov equation

$$
P=Y(F)^{T} P Y(F)+Z(F),
$$

where $Y(F)$ and $Z(F)$ are matrix functions of $F$ and $Z(F)$ is a symmetric matrix. Then

$$
\frac{\partial J}{\partial f_{i j}}=\frac{\partial}{\partial f_{i j}} \operatorname{tr}(P X)=2 \operatorname{tr}\left(\frac{\partial Y(F)^{T}}{\partial f_{i j}} P Y(F) S\right)+\operatorname{tr}\left(\frac{\partial Z(F)}{\partial f_{i j}} S\right),
$$

where $S$ satisfies the discrete Lyapunov equation

$$
S=Y(F) S Y(F)^{T}+X
$$

By using the above two lemmas we obtain the following result employed in the proof of Proposition 1 .

Lemma 3. Let $J(F)=\operatorname{tr}(P X)$ be a scalar function of the matrix variable $F$, where the symmetric matrix $X$ does not depend on $F$ and $P=P(F)$ satisfies the discrete Lyapunov equation

$$
P=(A+B F C)^{T} H^{T} P H(A+B F C)+Q+C^{T} F^{T} R F C+(A+B F C)^{T} W(A+B F C),
$$

where $A, B, C, H, Q, R$ and $W$ do not depend on $F$, and $Q, R$ and $W$ are symmetric matrices. Then

$$
\nabla_{F} J(F)=\frac{\partial}{\partial F} \operatorname{tr}(P X)=2\left[R F C+B^{T}\left(H^{T} P H+W\right)(A+B F C)\right] S C^{T},
$$

where $S$ satisfies the discrete Lyapunov equation

$$
S=H(A+B F C) S(A+B F C)^{T} H^{T}+X .
$$

Proof of Proposition 1. Let $F_{k}$ be a stabilizing periodic output feedback gain matrix. We deduce the expressions for the function and its gradient with respect to an arbitrary $F_{i}$. Let us replace $u_{k}=F_{k} C_{k} x_{k}$ in the performance index (2) to get

$$
J=E\left\{\sum_{k=0}^{\infty}\left[x_{k}^{T} \bar{Q}_{k} x_{k}\right]\right\}
$$


where $\bar{Q}_{k}=Q_{k}+C_{k}^{T} F_{k}^{T} R_{k} F_{k} C_{k}$ and $x_{k}$ satisfies the difference equation

$$
x_{k+1}=\bar{A}_{k} x_{k}
$$

where $\bar{A}_{k}=A_{k}+B_{k} F_{k} C_{k}$. It follows immediately that $x_{i}=\Phi_{\bar{A}}(i, 0) x_{0}$ and thus

$$
J=E\left\{\sum_{k=0}^{\infty}\left[x_{0}^{T} \Phi_{\bar{A}}^{T}(k, 0) \bar{Q}_{k} \Phi_{\bar{A}}(k, 0) x_{0}\right]\right\}
$$

We define

$$
P_{i}=\sum_{k=i}^{\infty} \Phi \frac{T}{A}(k, i) \bar{Q}_{k} \Phi_{\bar{A}}(k, i) .
$$

If $X_{0}=E\left\{x_{0} x_{0}^{T}\right\}$, then the performance index $J$ can be evaluated as

$$
J=\operatorname{tr}\left(P_{0} X_{0}\right) .
$$

It is easy to see that $P_{0}=P_{K}$ and that $P_{i}$ satisfies the DPLE (4). Further it is easy to show that each $P_{i}$ satisfies a discrete Lyapunov equation of the form

$$
P_{i}=\Phi \frac{T}{A}(i+K, i) P_{i} \Phi_{\bar{A}}(i+K, i)+\sum_{j=0}^{K-1} \Phi_{\bar{A}}^{T}(i+j, i) \bar{Q}_{i+j} \Phi_{\bar{A}}(i+j, i) .
$$

To compute the gradient of $J$ with respect to $F_{i}$, we rewrite $J$ as

$$
J=E\left\{\sum_{j=0}^{i-1}\left[x_{j}^{T} \bar{Q}_{j} x_{j}\right]\right\}+\operatorname{tr}\left(P_{i} X_{i}\right),
$$

where $X_{i}=E\left\{x_{i} x_{i}^{T}\right\}$ satisfies

$$
X_{i}=\Phi_{\bar{A}}(i, 0) X_{0} \Phi_{\bar{A}}(i, 0) .
$$

Notice that the first term in the expression of $J$ in (15) does not depend on $F_{i}$. It follows immediately that

$$
\frac{\partial J}{\partial F_{i}}=\frac{\partial}{\partial F_{i}} \operatorname{tr}\left(P_{i} X_{i}\right)
$$

By defining $H_{i}=\Phi_{\bar{A}}(i+K, i+1)$ and $W_{i}=\sum_{j=1}^{K-1} \Phi_{\bar{A}}^{T}(i+j, i+1) \bar{Q}_{i+j} \Phi_{\bar{A}}(i+j, i+1)$, we can rewrite the Lyapunov equation (14) in the form

$$
P_{i}=\bar{A}_{i}^{T} H_{i}^{T} P_{i} H_{i} \bar{A}_{i}+\bar{Q}_{i}+\bar{A}_{i}^{T} W_{i} \bar{A}_{i} .
$$

We can now apply Lemma 3 to get from (16) and (17)

$$
\frac{\partial J}{\partial F_{i}}=\frac{\partial}{\partial F_{i}} \operatorname{tr}\left(P_{i} X_{i}\right)=2\left(R_{i} F_{i} C_{i}+B_{i}^{T}\left(H_{i}^{T} P_{i} H_{i}+W_{i}\right) \bar{A}_{i}\right) S_{i} C_{i}^{T}
$$

where $S_{i}$ satisfies the discrete Lyapunov equation

$$
S_{i}=\Phi_{\bar{A}}(i+K, i) S_{i} \Phi_{\bar{A}}^{T}(i+K, i)+X_{i} .
$$

By using (4) we substitute first $P_{i}=\bar{A}_{i}^{T} P_{i+1} \bar{A}_{i}+\bar{Q}_{i}$ in the expression of the gradient (18). After straightforward matrix manipulations we further make the substitutions $\bar{A}_{i} H_{i}=H_{i+1} \bar{A}_{i+1}$ and $W_{i}+H_{i}^{T} \bar{Q}_{i} H_{i}=\bar{Q}_{i+1}+\bar{A}_{i+1}^{T} W_{i+1} \bar{A}_{i+1}$ to obtain with the help of (17)

$$
\frac{\partial J}{\partial F_{i}}=2\left(R_{i} F_{i} C_{i}+B_{i}^{T} P_{i+1} \bar{A}_{i}\right) S_{i} C_{i}^{T} .
$$

It can be verified that the symmetric matrices $S_{i}, i=0, \ldots, K-1$, satisfy the DPLE (5). 